\title{
GLOBAL EXISTENCE FOR NONLINEAR SYSTEM OF WAVE EQUATIONS IN 3-D DOMAINS
}

Abstract. We study the initial-boundary problem for a nonlinear system of wave equations with Hamilton structure under Dirichlet's condition. We use the local-in-time Strichartz estimates from [Burq et al., J. Amer. Math. Soc. 21 (2008), 831-845], Morawetz-Pohožaev's identity derived in [Miao and Zhu, Nonlinear Anal. 67 (2007), 3136-3151], and an a priori estimate of the solutions restricted to the boundary to show the existence of global and unique solutions.

1. Introduction. Numerous works have been devoted to the study of Cauchy problems for the semilinear wave equation

$$
\left\{\begin{array}{l}
u_{t t}-\Delta u+u|u|^{p-1}=0, \quad(t, x) \in \mathbb{R} \times \mathbb{R}^{d}, \\
u(0, x)=\phi(x), \quad \partial_{t} u(0, x)=\psi(x), \quad x \in \mathbb{R}^{d},
\end{array}\right.
$$

where $p_{c} \equiv \frac{d+2}{d-2}$ corresponds to the energy critical growth of the nonlinearity.

For $d=3$ and $1<p<p_{c}$, Jörgens [9] proved the existence and uniqueness of global-in-time smooth solutions of problem (1.1). In the critical case $p=p_{c}$, Rauch [12] proved an analogous result for smooth solutions under a small energy assumption, i.e. for

$$
E(\phi, \psi)=\int_{\mathbb{R}^{d}}\left(\frac{1}{2}|\nabla \phi|^{2}+\frac{1}{2}|\psi|^{2}+\frac{1}{p+1}|\phi|^{p+1}\right) d x \ll 1 .
$$

Next, Struwe [19] established the existence and uniqueness of a global smooth solution to (1.1), $u(t, x) \in C^{2}\left(\mathbb{R} \times \mathbb{R}^{3}\right)$ for radial initial data $\phi(x)=\phi(|x|) \in$ $C^{3}\left(\mathbb{R}^{3}\right), \psi(x)=\psi(|x|) \in C^{2}\left(\mathbb{R}^{3}\right)$. Finally, Grillakis [8] removed Struwe's radial assumption using Morawetz's estimate.

2000 Mathematics Subject Classification: 35L05, 35L70.

Key words and phrases: nonlinear wave equations, Dirichlet boundary condition. 
For finite energy solutions, Ginibre and Velo [6, 7] obtained the global existence and uniqueness of solution to (1.1) when $1<p<p_{c}$. Later on, Shatah and Struwe [15] proved the same result for finite energy solutions in the critical case $p=p_{c}$. We refer to the excellent book [10] for a comprehensive discussion of the history and results on problem $(1.1)$, ranging from smooth solutions to finite energy ones and from low dimensions to higher dimensions including well-posedness and scattering theory.

A study of initial-value problems for the energy critical problem posed in the exterior of a smooth, compact and strictly convex obstacle $\Theta \subset \mathbb{R}^{3}$ is due to Smith and Sogge [16], who considered the problem

$$
\left\{\begin{array}{l}
u_{t t}-\Delta u+u^{5}=0, \quad(t, x) \in \mathbb{R}_{+} \times\left(\mathbb{R}^{3} \backslash \Theta\right), \\
u(0, x)=\phi(x) \in C^{\infty}\left(\mathbb{R}^{3} \backslash \Theta\right), \\
\partial_{t} u(0, x)=\psi(x) \in C^{\infty}\left(\mathbb{R}^{3} \backslash \Theta\right), \\
u(t, x)=0, \quad x \in \partial \Theta,
\end{array}\right.
$$

with $\phi$ and $\psi$ satisfying a necessary compatibility condition. They explored several basic Strichartz type inequalities analogous to those for Cauchy problems by exploiting $L^{2}$ continuity of certain operators. As a consequence, they proved the existence and uniqueness of global smooth solutions to (1.2).

Those results were extended by Burq, Lebeau and Planchon [1], who proved the global existence of solutions for the same semilinear wave equation posed on a general smooth compact domain with the Dirichlet boundary condition. The main ingredient in their proof was a local-in-time Strichartz type estimate without loss of derivative, which is based on the $L^{p}$ estimates for the associated spectral projector on manifolds obtained by Smith and Sogge [17. This local-in-time space-time estimate is sufficient for the global existence theory. Shortly after, Burq and Planchon [2] proved the same result under the Neumann boundary condition; for this, they developed a meticulous analysis to control the behavior of the solution on the boundary due to the failure of the uniform Lopatinski condition.

In this article, we are going to prove analogous results for the repulsive nonlinear system of wave equations

$$
\left\{\begin{array}{l}
u_{t t}-\Delta u+\left(|u|^{4}+|v|^{2}\right) u=0, \\
v_{t t}-\Delta v+\left(|v|^{4}+|u|^{2}\right) v=0 .
\end{array}\right.
$$

This system appears naturally in the description of the interaction of two distinct scalar fields, where the constants have been assumed to be 1 . More general systems similar to (1.3) describe the motion of charged mesons in an electromagnetic field. More details on the physical background can be found in [13] and in the references therein. 
Let $\Omega \subset \mathbb{R}^{3}$ be a bounded domain with smooth boundary $\partial \Omega$, and $-\Delta_{D}$ the corresponding Dirichlet-Laplacian. We consider the coupled wave equations 1.3 for $(t, x) \in \mathbb{R}_{t} \times \Omega$, supplemented with the initial data

$$
\begin{cases}\left.u\right|_{t=0}=\phi_{1}, & \left.u_{t}\right|_{t=0}=\psi_{1}, \\ \left.v\right|_{t=0}=\phi_{2}, & \left.v_{t}\right|_{t=0}=\psi_{2},\end{cases}
$$

and subject to Dirichlet's boundary condition

$$
\left.u\right|_{\partial \Omega}=\left.v\right|_{\partial \Omega}=0 .
$$

We assume that $u(t, x)$ and $v(t, x)$ are real-valued functions for simplicity (so the initial data are real functions).

REMARK 1.1. As in [11, the nonlinear terms in equations 1.3 may have a more general form. Given $F(\lambda, \mu)$, we denote

$$
F_{1}(\lambda, \mu)=\frac{\partial F(\lambda, \mu)}{\partial \lambda} \quad \text { and } \quad F_{2}(\lambda, \mu)=\frac{\partial F(\lambda, \mu)}{\partial \mu} .
$$

Then the inhomogeneous terms in 1.3 can be replaced respectively with

$$
F_{1}\left(|u|^{2},|v|^{2}\right) u \text { and } F_{2}\left(|u|^{2},|v|^{2}\right) v
$$

where $F\left(|u|^{2},|v|^{2}\right)$ satisfies conditions from [11]; our method can be adapted to handle this general case.

First, notice that problem (1.3)-(1.5) satisfies the following conservation of energy (see [11] for details):

$$
\begin{aligned}
E(u, v)(t) & =\int_{\Omega}\left(\frac{1}{2}\left(\left|\nabla_{t, x} u\right|^{2}+\left|\nabla_{t, x} v\right|^{2}\right)+\frac{1}{6}\left(|u|^{6}+|v|^{6}\right)+\frac{1}{2}|u|^{2}|v|^{2}\right) d x \\
& =E(u, v)(0),
\end{aligned}
$$

where $\nabla_{t, x}:=\left(\partial_{t}, \nabla_{x}\right)$.

Our main result can be stated as follows.

TheOREM 1.1. For any initial datum $\left(\phi_{j}, \psi_{j}\right) \in H_{0}^{1}(\Omega) \times L^{2}(\Omega), j=$ 1,2 , there exists a unique global solution $(u, v)$ to problem $1.3-1.5$ in the space

$$
\begin{aligned}
\mathcal{X}= & C^{0}\left(\mathbb{R}_{t} ; H_{0}^{1}(\Omega) \times H_{0}^{1}(\Omega)\right) \cap C^{1}\left(\mathbb{R}_{t} ; L^{2}(\Omega) \times L^{2}(\Omega)\right) \\
& \cap L_{\text {loc }}^{5}\left(\mathbb{R}_{t} ; L^{10}(\Omega) \times L^{10}(\Omega)\right) .
\end{aligned}
$$

The local existence and uniqueness of solution to $1.3-(1.5)$ is based on the Strichartz estimate. To get the existence of global and unique solutions, we need to deduce a priori boundary estimates following [1]. The idea and underlying techniques come from [1] but we need a careful analysis of the nonlinear interacting waves in (1.3) in order to get a global solution.

This paper is organized as follows. We introduce some basic notation at the end of this section. In Section 2, the existence and uniqueness of local-in-time 
solutions is sketched by a fixed-point argument. In Section 3, we prove a priori space-time $L^{2}$ boundedness for the exterior normal derivative of the finite energy solution to the system on the boundary. Section 4 is devoted to proving the global existence of $(u, v)$ by an argument based on the Morawetz-Pohožaev identity derived in [11]; this completes the proof of Theorem 1.1.

We define the norm for the vector $(u, v)$ in the product space $L^{5}\left(I ; L^{10}(\Omega)\right.$ $\left.\times L^{10}(\Omega)\right)$ by

$$
\|(u, v)\|_{L^{5}\left(I ; L^{10}(\Omega) \times L^{10}(\Omega)\right)}:=\|u\|_{L^{5}\left(I ; L^{10}(\Omega)\right)}+\|v\|_{L^{5}\left(I ; L^{10}(\Omega)\right)} ;
$$

in the same way we define the norms $\|(u, v)\|_{C^{0}\left(I ; H_{0}^{1}(\Omega) \times H_{0}^{1}(\Omega)\right)}$ etc., where $I$ is a time interval.

We shall also write the norms as $\|(u, v)\|_{L^{5}\left(I ; L^{10}(\Omega)\right)}$ and $\|(u, v)\|_{C^{0}\left(I ; H_{0}^{1}(\Omega)\right)}$ etc. for short. Unimportant constants in inequalities may vary and we denote them by a capital letter $C$ for convenience, unless further notation is specified. Finally, for harmonic analysis tools with applications to wave equations, we refer the reader to [10].

2. Local existence of solutions. Here, we use the following Strichartz estimate which is proved in [1].

Proposition 2.1 (Burq-Lebeau-Planchon [2, Prop. 3.1]). If $u, f_{1}, f_{2}$ satisfy

$$
\partial_{t}^{2} u-\Delta_{D} u=f_{1}+f_{2},\left.\quad u\right|_{\partial \Omega}=0,\left.\quad u\right|_{t=0}=\phi,\left.\quad \partial_{t} u\right|_{t=0}=\psi,
$$

then

$$
\begin{aligned}
& \|u\|_{L^{5}\left((0,1) ; W_{0}^{3 / 10,5}(\Omega)\right)}+\|u\|_{C^{0}\left((0,1) ; H_{0}^{1}(\Omega)\right)}+\left\|u_{t}\right\|_{C^{0}\left((0,1) ; L^{2}(\Omega)\right)} \\
& \leq C\left(\|\phi\|_{H_{0}^{1}(\Omega)}+\|\psi\|_{L^{2}(\Omega)}+\left\|f_{1}\right\|_{L^{5 / 4}\left((0,1) ; W^{7 / 10,5 / 4}(\Omega)\right)}+\left\|f_{2}\right\|_{L^{1}\left((0,1) ; L^{2}(\Omega)\right)}\right) .
\end{aligned}
$$

This section is devoted to the proof of the local existence of solutions to the boundary value problem (1.3)- 1.5$)$. It is well known that the problem is equivalent to the following integral equations:

$$
\begin{aligned}
u(t)= & \cos t \sqrt{-\Delta_{D}} \phi_{1}+\frac{\sin t \sqrt{-\Delta_{D}}}{\sqrt{-\Delta_{D}}} \psi_{1} \\
& -\int_{0}^{t} \frac{\sin (t-s) \sqrt{-\Delta_{D}}}{\sqrt{-\Delta_{D}}}\left(\left(|u|^{4}+|v|^{2}\right) u\right)(s) d s, \\
v(t)= & \cos t \sqrt{-\Delta_{D}} \phi_{2}+\frac{\sin t \sqrt{-\Delta_{D}}}{\sqrt{-\Delta_{D}}} \psi_{2} \\
& -\int_{0}^{t} \frac{\sin (t-s) \sqrt{-\Delta_{D}}}{\sqrt{-\Delta_{D}}}\left(\left(|v|^{4}+|u|^{2}\right) v\right)(s) d s .
\end{aligned}
$$

To show the existence of local-in-time solutions of system (2.1a)-(2.1b), we estimate its nonlinear terms by using Strichartz's estimates from Propo- 
sition 2.1. Without loss of generality, we only deal with equation (2.1a). Obviously, we have

$$
\begin{aligned}
\left\|\left(|u|^{4}+|v|^{2}\right) u\right\|_{L^{5 / 4}\left(J ; W^{7 / 10,5 / 4}(\Omega)\right)} & \\
& \leq\left\|u^{5}\right\|_{L^{5 / 4}\left(J ; W^{7 / 10,5 / 4}(\Omega)\right)}+\left\||v|^{2} u\right\|_{L^{5 / 4}\left(J ; W^{7 / 10,5 / 4}(\Omega)\right)}
\end{aligned}
$$

As in [1], the first term on the right hand side is bounded, up to a constant, by

$$
\|u\|_{L^{5}\left(J ; L^{10}(\Omega)\right)}^{4}\|u\|_{L^{\infty}\left(J ; L^{6}(\Omega)\right)}^{3 / 10}\|u\|_{L^{\infty}\left(J ; H^{1}(\Omega)\right)}^{7 / 10} .
$$

For the second term, the Hölder inequality and the compactness of $\bar{\Omega}$ yield $(2.2)$

$$
\begin{aligned}
\left\|v^{2} u\right\|_{L^{5 / 4}\left(J ; L^{30 / 17}(\Omega)\right)} & \leq\|\| v^{2}\left\|_{L^{5 / 2}(\Omega)}\right\| u\left\|_{L^{6}(\Omega)}\right\|_{L^{5 / 4}(J)} \\
& =\|\| v\left\|_{L^{5}(\Omega)}^{2}\right\| u\left\|_{L^{6}(\Omega)}\right\|_{L^{5 / 4}(J)} \\
& \leq C\|\| v\left\|_{L^{10}(\Omega)}^{2}\right\| u\left\|_{L^{6}(\Omega)}\right\|_{L^{5 / 4}(J)} \\
& \leq C\|v\|_{L^{5}\left(J ; L^{10}(\Omega)\right)}^{2}\|u\|_{L^{\infty}\left(J ; L^{6}(\Omega)\right)}|J|^{2 / 5} .
\end{aligned}
$$

On the other hand, by Leibniz's rule and triangle inequality, we get $\left\|\nabla\left(v^{2} u\right)\right\|_{L^{5 / 4}\left(J ; L^{10 / 9}(\Omega)\right)} \leq 2\|u v \nabla v\|_{L^{5 / 4}\left(J ; L^{10 / 9}(\Omega)\right)}+\left\|v^{2} \nabla u\right\|_{L^{5 / 4}\left(J ; L^{10 / 9}(\Omega)\right)}$. Since

$$
\begin{aligned}
\|v u \nabla v\|_{L^{10 / 9}(\Omega)} & \leq\|v u\|_{L^{5 / 2}(\Omega)}\|\nabla v\|_{L^{2}(\Omega)} \leq C\|u\|_{L^{5}(\Omega)}\|v\|_{L^{5}(\Omega)}\|v\|_{H_{0}^{1}(\Omega)} \\
& \leq C\|v\|_{H_{0}^{1}}\left(\|u\|_{L^{10}(\Omega)}^{2}+\|v\|_{L^{10}(\Omega)}^{2}\right),
\end{aligned}
$$

we have

$$
\begin{aligned}
& \|u v \nabla v\|_{L^{5 / 4}\left(J ; L^{10 / 9}(\Omega)\right)} \leq C\|\| v\left\|_{H_{0}^{1}}\left(\|u\|_{L^{10}(\Omega)}^{2}+\|v\|_{L^{10}(\Omega)}^{2}\right)\right\|_{L^{5 / 4}(J)} \\
& \leq C|J|^{2 / 5}\|v\|_{L^{\infty}\left(J ; H_{0}^{1}(\Omega)\right)}\left(\|u\|_{L^{5}\left(J ; L^{10}(\Omega)\right)}+\|v\|_{L^{5}\left(J ; L^{10}(\Omega)\right)}\right)^{2} .
\end{aligned}
$$

Similarly we can deduce that

$$
\left\|v^{2} \nabla u\right\|_{L^{5 / 4}\left(J ; L^{10 / 9}(\Omega)\right)} \leq C\|v\|_{L^{5}\left(J ; L^{10}(\Omega)\right)}^{2}\|u\|_{L^{\infty}\left(J ; H_{0}^{1}(\Omega)\right)}|J|^{2 / 5} .
$$

Combining 2.2 and $(2.3)$, we arrive at

$$
\left\|v^{2} u\right\|_{L^{5 / 4}\left(J ; L^{30 / 17}(\Omega)\right)} \leq C\|(u, v)\|_{L^{5}\left(J: L^{10}(\Omega)\right)}^{2}\|(u, v)\|_{L^{\infty}\left(J ; L^{6}(\Omega)\right)}|J|^{2 / 5}
$$

and

$$
\begin{aligned}
& \left\|\nabla\left(v^{2} u\right)\right\|_{L^{5 / 4}\left(J ; L^{10 / 9}(\Omega)\right)} \\
& \quad \leq C\|(u, v)\|_{L^{5}\left(J ; L^{10}(\Omega)\right)}^{2}\|(u, v)\|_{L^{\infty}\left(J ; H_{0}^{1}(\Omega)\right)}|J|^{2 / 5} .
\end{aligned}
$$

Interpolating between (2.4) and $(2.5)$ yields

$$
\begin{aligned}
\left\||v|^{2} u\right\|_{L^{5 / 4}\left(J ; W^{7 / 10,5 / 4}(\Omega)\right)} \leq & C\|(u, v)\|_{L^{5}\left(J: L^{10}(\Omega)\right)}^{2}\|(u, v)\|_{L^{\infty}\left(J ; L^{6}(\Omega)\right)}^{3 / 10} \\
& \times\|(u, v)\|_{L^{\infty}\left(J ; H_{D}^{1}(\Omega)\right)}^{7 / 10}|J|^{2 / 5}
\end{aligned}
$$


Thus, we conclude that, with $|J|<1$, we have

$$
\begin{aligned}
\left\|\left(|u|^{4}+|v|^{2}\right) u\right\|_{L^{5 / 4}\left(J ; W^{7 / 10,5 / 4}(\Omega)\right)} & \\
\leq & C\left(\|(u, v)\|_{L^{5}\left(J ; L^{10}(\Omega)\right)}^{4}+\|(u, v)\|_{L^{5}\left(J ; L^{10}(\Omega)\right)}^{2}\right) \\
& \times\|(u, v)\|_{L^{\infty}\left(J ; H_{0}^{1}(\Omega)\right)}^{7 / 10}\|(u, v)\|_{L^{\infty}\left(J ; L^{6}(\Omega)\right)}^{3 / 10},
\end{aligned}
$$

and similarly

$$
\begin{aligned}
&\left\|\left(|v|^{4}+|u|^{2}\right) v\right\|_{L^{5 / 4}\left(J ; W^{7 / 10,5 / 4}(\Omega)\right)} \\
& \leq C\left(\|(u, v)\|_{L^{5}\left(J ; L^{10}(\Omega)\right)}^{4}+\|(u, v)\|_{L^{5}\left(J ; L^{10}(\Omega)\right)}^{2}\right) \\
& \times\|(u, v)\|_{L^{\infty}\left(J ; H_{0}^{1}(\Omega)\right)}^{7 / 10}\|(u, v)\|_{L^{\infty}\left(J ; L^{6}(\Omega)\right)}^{3 / 0}
\end{aligned}
$$

Here, we have dropped the factor $|J|^{2 / 5}$ in the estimates 2.6 and 2.7 ) since the nonlinearity is a combination of a subcritical growth and a critical one. In contrast to the subcritical case, the quantity $|J|^{2 / 5}$ in $(2.4)$ and $(2.5)$ does not help to produce a contracting factor in the fixed-point argument. Instead, to get a contraction map, we have to use a space-time integral.

Now, we consider the existence of local-in-time solutions. Let $(a, b)^{T}$ denote the transpose of the vector $(a, b)$. We define an operator $\mathcal{T}$ by

$$
\mathcal{T}(U(t), V(t)):=\mathcal{W}(t)(U(0), V(0))-\int_{0}^{t} \mathcal{W}(t-s) F(u, v)(s) d s
$$

where

$$
\begin{aligned}
& \mathcal{W}(t)=\left(\begin{array}{cc}
\cos t \sqrt{-\Delta_{D}} & \frac{\sin t \sqrt{-\Delta_{D}}}{\sqrt{-\Delta_{D}}} \\
-\sqrt{-\Delta_{D}} \sin t \sqrt{-\Delta_{D}} & \cos t \sqrt{-\Delta_{D}}
\end{array}\right), \\
& U(t)=\left(u(t), \partial_{t} u(t)\right)^{T}, \quad V(t)=\left(v(t), \partial_{t} v(t)\right)^{T}, \\
& F(u, v)(t)=\left(\begin{array}{cc}
0 & 0 \\
\left(|u|^{4}+|v|^{2}\right) u(t) & \left(|v|^{4}+|u|^{2}\right) v(t)
\end{array}\right) .
\end{aligned}
$$

The wave group $\mathcal{W}(t)$ allows one to write problem 1.3$]-1.5)$ in the following way:

$$
(U(t), V(t))=\mathcal{T}(U(t), V(t)) .
$$

This suggests that the solution is a fixed point of some operator in a suitable function space. To perform the fixed-point argument, we define the operator

$$
\begin{aligned}
T(u, v)(t)= & K^{\prime}(t)\left(\phi_{1}, \phi_{2}\right)+K(t)\left(\psi_{1}, \psi_{2}\right) \\
& -\int_{0}^{t} K(t-s)\left(\left(|u|^{4}+|v|^{2}\right) u,\left(|v|^{4}+|u|^{2}\right) v\right)(s) d s,
\end{aligned}
$$


where

$$
K(t)=\frac{\sin t \sqrt{-\Delta_{D}}}{\sqrt{-\Delta_{D}}} .
$$

We have the following theorem concerning the existence and uniqueness of local-in-time solutions to problem (1.3)-(1.5).

Theorem 2.1. Let $\left(\phi_{j}, \psi_{j}\right) \in H_{0}^{1}(\Omega) \times L^{2}(\Omega), j=1,2$, let $J \ni 0$ be an interval and suppose $\left\|\left(\phi_{1}, \phi_{2}\right)\right\|_{H_{0}^{1}(\Omega)}+\left\|\left(\psi_{1}, \psi_{2}\right)\right\|_{L^{2}(\Omega)} \leq M:=C E(u, v)(0)$. Then there is an $\epsilon=\epsilon(M)$ such that if

$$
\left\|K^{\prime}(t)\left(\phi_{1}, \phi_{2}\right)+K(t)\left(\psi_{1}, \psi_{2}\right)\right\|_{L^{5}\left(J ; L^{10}(\Omega)\right)} \leq \epsilon,
$$

then there exists a unique solution $(u, v)$ in $J \times \Omega$ with $(u, v) \in C\left(J ; H_{0}^{1}(\Omega) \times\right.$ $\left.H_{0}^{1}(\Omega)\right) \cap C^{1}\left(J ; L^{2}(\Omega) \times L^{2}(\Omega)\right)$. Moreover, the flow map generated by the system is Lipschitz continuous in $C\left(J ; H_{0}^{1}(\Omega) \times H_{0}^{1}(\Omega)\right) \cap C^{1}\left(J ; L^{2}(\Omega) \times\right.$ $\left.L^{2}(\Omega)\right)$.

Proof. We first set

$$
\begin{aligned}
& \mathcal{X}(J)=\left\{(u, v) \in C^{0}\left(J ; H_{0}^{1}(\Omega) \times H_{0}^{1}(\Omega)\right)\right. \cap L^{5}\left(J ; L^{10}(\Omega) \times L^{10}(\Omega)\right): \\
&\left.\|(u, v)\|_{L^{5}\left(J ; L^{10}(\Omega)\right)} \leq \eta,\|(u, v)\|_{C\left(J ; H_{0}^{1}(\Omega)\right)} \leq 4 M\right\}
\end{aligned}
$$

with $\eta$ to be determined later.

By Strichartz's estimates from Proposition 2.1 and the nonlinear estimates (2.6), (2.7), we have

$$
\|T(u, v)\|_{C\left(J ; H_{0}^{1}(\Omega)\right)} \leq M+C 4 M\left(\eta^{3}+\eta\right) \eta .
$$

Take $\eta \in(0,1)$ such that $C\left(\eta^{3}+\eta\right)<1 / 4 ;$ then

$$
\|T(u, v)\|_{C\left(J ; H_{0}^{1}(\Omega)\right)} \leq M+M \leq 4 M .
$$

On the other hand, using Strichartz's estimates again, we have

$$
\|T(u, v)\|_{L^{5}\left(J ; L^{10}(\Omega)\right)} \leq \epsilon+C 4 M\left(\eta^{3}+\eta\right) \eta .
$$

Taking $\epsilon=\eta / 2$ and $\eta \in(0,1)$ (or smaller if necessary) so that $C 4 M\left(\eta^{3}+\eta\right)$ $<1 / 2$, we get

$$
\|T(u, v)\|_{L^{5}\left(J ; L^{10}(\Omega)\right)} \leq \eta .
$$

These imply that $T$ maps $\mathcal{X}(J)$ to itself. Next, we define a metric $d(\cdot, \cdot)=$ $\|\cdot-\cdot\|_{C\left(J ; H_{0}^{1}(\Omega)\right)}+\|\cdot-\cdot\|_{L^{5}\left(J ; L^{10}(\Omega)\right)}$ on $\mathcal{X}(J)$; then $\mathcal{X}(J)$ becomes a complete metric space. By Strichartz's estimate, for any two solutions $\left(u_{1}, v_{1}\right),\left(u_{2}, v_{2}\right)$ $\in \mathcal{X}(J)$ with the same initial data, we have

$$
\begin{aligned}
d\left(T\left(u_{1}, v_{1}\right), T\left(u_{2}, v_{2}\right)\right) & \leq C\left(\eta+\eta^{3}\right) 4 M d\left(\left(u_{1}, v_{1}\right),\left(u_{2}, v_{2}\right)\right) \\
& \leq \frac{1}{2} d\left(\left(u_{1}, v_{1}\right),\left(u_{2}, v_{2}\right)\right),
\end{aligned}
$$


which indicates that $T$ is a contraction map on $\mathcal{X}(J)$ and we get the local well-posedness for problem (1.3)-(1.5) by Banach's fixed-point theorem and Strichartz's estimate. Thus, the proof of Theorem 2.1 is complete.

3. A priori estimate. In order to obtain the global existence of solution, we have to control the behavior of a certain quantity on the boundary, following [1] and [11, in order to exclude the concentration effect of the potential energy. Since mild solutions of 1.3 1.5 are limits of smooth solutions, we can assume that $(u, v)$ are regular enough in our calculations below.

Suppose $t_{0}$ is the lifespan of the solution to $1.3-1.5$ in $\mathcal{X}(J)$, with $J=\left(0, t_{0}\right)$. First, we move $t_{0}$ to 0 by translation invariance of system 1.3$)$. With $T<S<0$, our notation below is taken from [1] and [11]. We define the following quantities:

$$
\begin{aligned}
& Q= \frac{\left|\nabla_{t, x} u\right|^{2}+\left|\nabla_{t, x} v\right|^{2}+|u|^{2}|v|^{2}}{2} \\
&+\frac{1}{t}\left(u_{t} x \cdot \nabla u+v_{t} x \cdot \nabla v\right)+\frac{1}{6}\left(|u|^{6}+|v|^{6}\right), \\
& P= \frac{1}{2}\left(\left|u_{t}\right|^{2}+\left|v_{t}\right|^{2}-|\nabla u|^{2}-|\nabla v|^{2}-\frac{1}{3}\left(|u|^{6}+|v|^{6}\right)-|u|^{2}|v|^{2}\right) \frac{x}{t} \\
&+\left(\frac{u}{t}+u_{t}+\frac{x \cdot \nabla u}{t}\right) \nabla u+\left(\frac{v}{t}+v_{t}+\frac{x \cdot \nabla v}{t}\right) \nabla v, \\
& \Omega_{T}^{S}= {[T, S] \times \Omega, \quad \partial \Omega_{T}^{S}=[T, S] \times \partial \Omega, } \\
& D_{T}=\{x:|x|<-T\}, \quad M_{T}^{S}=\{(x, t):|x|=-t, T<t<S\}, \\
& K_{T}^{S}=\{(x, t):|x|<-t, T<t<S\}, \\
& \partial K_{T}^{S}= D_{T} \cup D_{S} \cup M_{T}^{S}, \quad \\
& e(u, v)=\left(-u_{t} \nabla u-v_{t} \nabla v, \quad \frac{1}{2}\left(\left|\nabla_{t, x} u\right|^{2}+\left|\nabla_{t, x} v\right|^{2}\right)+\frac{1}{6}\left(|u|^{6}+|v|^{6}\right)+\frac{1}{2}|u|^{2}|v|^{2}\right), \\
&\left.\operatorname{Flux}_{(u, v}, M_{T}^{S}\right)=\int_{M_{T}^{S} \cap \Omega_{T}^{S}}\langle e(u, v), \vec{\nu}\rangle d \sigma(x, t) \\
&=\frac{1}{2 \sqrt{2}}{ }_{M_{T}^{S} \cap \Omega_{T}^{S}}\left(\left|\frac{x}{|x|} u_{t}-\nabla u\right|^{2}+\left|\frac{x}{|x|} v_{t}-\nabla v\right|^{2}\right. \\
&\left.+|u|^{2}|v|^{2}+\frac{1}{3}\left(|u|^{6}+|v|^{6}\right)\right) d \sigma(x, t) \geq 0,
\end{aligned}
$$


where $\vec{\nu}=\frac{1}{\sqrt{2}}(x /|x|, 1)$ is the exterior normal vector to $M_{T}^{S}, d \sigma(x, t)$ is the induced space-time measure and there is no flux across $\partial \Omega_{T}^{S}$ due to the boundary condition. As in [11], we define the local energy by

$$
\begin{aligned}
& E_{\mathrm{loc}}\left(u, v, D_{T}\right) \\
:= & \int_{D_{T} \cap \Omega}\left[\frac{1}{2}\left(\left|\nabla_{t, x} u\right|^{2}+\left|\nabla_{t, x} v\right|^{2}+|u|^{2}|v|^{2}\right)+\frac{1}{6}\left(|u|^{6}+|v|^{6}\right)\right](x, T) d x .
\end{aligned}
$$

Due to the finite speed of propagation, we have

$$
E_{\mathrm{loc}}\left(u, v, D_{T}\right)=E_{\mathrm{loc}}\left(u, v, D_{S}\right)+\operatorname{Flux}\left(u, v, M_{T}^{S}\right) .
$$

Therefore $E_{\mathrm{loc}}\left(u, v, D_{T}\right)$ and $E_{\mathrm{loc}}\left(u, v, D_{S}\right)$ converge to the same limit as $T, S \nearrow 0^{-}$since $E_{\mathrm{loc}}\left(u, v, D_{T}\right)$ is nonincreasing and bounded below. Hence $\operatorname{Flux}\left(u, v, M_{T}^{S}\right)$ tends to zero as $T \nearrow 0^{-}$.

First, we deduce an $L^{2}$ space-time estimate for the exterior normal derivative of $(u, v)$ on the boundary.

Lemma 3.1. Suppose $(u, v)$ is a finite energy solution of problem (1.3)(1.5), and $\vec{n}(x)$ is an exterior normal vector to $\partial \Omega$. Then

$$
\left\|\frac{\partial u}{\partial \vec{n}}\right\|_{L^{2}\left(\left(0, t_{0}\right) \times \partial \Omega\right)}+\left\|\frac{\partial v}{\partial \vec{n}}\right\|_{L^{2}\left(\left(0, t_{0}\right) \times \partial \Omega\right)} \leq C E(u, v)^{1 / 2} .
$$

Proof. Without loss of generality, we estimate $\partial u / \partial \vec{n}$ only. Similarly to [1], considering a smooth vector field $\Gamma \in C^{\infty}(\Omega ; T \Omega)$ whose restriction to $\partial \Omega$ is equal to $\partial / \partial \vec{n}$, where $T \Omega$ is the tangent bundle over $\Omega$ and $0<T<t_{0}$, we integrate by parts to get

$$
\begin{aligned}
\int_{0}^{T} \int_{\Omega}\left[\partial_{t}^{2}-\Delta, \Gamma\right] u \cdot u d x d t \\
=\int_{0}^{T} \int_{\Omega}\left(\partial_{t}^{2}-\Delta\right) \Gamma(u) u d x d t-\int_{0}^{T} \int_{\Omega} \Gamma\left(-|u|^{4} u-v^{2} u\right) u d x d t \\
=\int_{0}^{T} \int_{\partial \Omega}\left|\frac{\partial u}{\partial \vec{n}}\right|^{2} d \varrho(x) d t+\left.\left[\int_{\Omega} u \partial_{t} \Gamma(u) d x-\int_{\Omega} \Gamma(u) u_{t} d x\right]\right|_{0} ^{T} \\
\quad+\int_{0}^{T} \int_{\Omega}\left[\Gamma(u)\left(-u^{5}-v^{2} u\right)+\Gamma\left(u^{5}+v^{2} u\right) u\right] d x d t \\
=I+I I+I I I,
\end{aligned}
$$

where $d \varrho(x)$ is the surface measure on $\partial \Omega$.

In order to control $I$, we first apply integration by parts and the CauchySchwarz inequality to see that the second term $I I$ can be bounded by $E(u, v)$. 
Secondly, direct computation gives

$$
\begin{aligned}
I I I & =\int_{0}^{T} \int_{\Omega}\left(4 u^{5} \Gamma(u)+u^{2} \Gamma\left(v^{2}\right)\right) d x d t \\
& =\int_{0}^{T} \int_{\Omega} \frac{2}{3} \Gamma\left(u^{6}\right) d x d t+\int_{0}^{T} \int_{\Omega} 2 u^{2} v \Gamma(v) d x d t .
\end{aligned}
$$

The first term in $I I I$ is bounded by $E(u, v)$ (see [1, Proposition 3.2]). By Hölder's inequality, the second one is controlled by

$$
\left(\int_{0}^{T} \int_{\Omega}|\Gamma(v)|^{2}\right)^{1 / 2}\left(\int_{0}^{T} \int_{\Omega}|u|^{6}\right)^{1 / 3}\left(\int_{0}^{T} \int_{\Omega}|v|^{6}\right)^{1 / 6} \leq C E(u, v)^{1 / 2+1 / 3+1 / 6},
$$

where we have used

$$
|\Gamma(v)|=\left|\sum_{j} a_{j}(x) \frac{\partial}{\partial x_{j}} v\right| \leq|a||\nabla v| \leq \sup _{x \in \Omega}|a||\nabla v| .
$$

Summing up the above estimates and observing that $\left[\partial_{t}^{2}-\Delta, \Gamma\right]=$ $[-\Delta, \Gamma]: H_{0}^{1}(\Omega) \rightarrow H^{-1}(\Omega)$ is bounded (because the commutator is of order 2$)$, we conclude that

$$
\left\|\frac{\partial u}{\partial \vec{n}}\right\|_{L^{2}\left(\left(0, t_{0}\right) \times \partial \Omega\right)} \leq C E(u, v)^{1 / 2} .
$$

A similar argument gives

$$
\left\|\frac{\partial v}{\partial \vec{n}}\right\|_{L^{2}\left(\left(0, t_{0}\right) \times \partial \Omega\right)} \leq C E(u, v)^{1 / 2} .
$$

which completes the proof of Lemma 3.1.

REMARK 3.1. There is a connection between Lemma3.1 and the uniform Lopatinski condition for mixed Cauchy problems of hyperbolic equations. The Cauchy-Dirichlet problems satisfy the uniform Lopatinski condition for almost trivial reasons since the Lopatinski determinant is constant. However, the Neumann boundary problems violate the uniform Lopatinski condition due to the possibility of degeneration of the Lopatinski determinant on an imbedded surface in the cotangent bundle of $\partial \Omega$. See, for example, [2] and [3] for more details.

4. Global existence of solutions. The key role in proving Theorem 1.1 is played by the following estimate which indicates the classical nonconcentration effect on small time slices:

$$
\int_{D_{T} \cap \Omega}\left(|u|^{6}+|v|^{6}\right)(T, x) d x \rightarrow 0 \quad \text { as } T \nearrow 0^{-} .
$$


To prove this relation, we will employ the Morawetz-Pohožaev identity according to [11]. Next, we localize our estimates in a small space-time light cone in the same way as in [14, and then use Lemma 3.1 to control the boundary terms.

Integrating the following identity obtained in [1] over $K_{T}^{S} \cap \Omega_{T}^{S}:=D$,

$$
\operatorname{div}_{x, t}\left(-t P, t Q+u_{t} u+v_{t} v\right)+\frac{1}{3}\left(|u|^{6}+|v|^{6}\right)=0,
$$

one has

$$
\begin{aligned}
\int_{D_{S} \cap \Omega}\left(S Q+u_{t} u+v_{t} v\right) & (S, x) d x-\int_{D_{T} \cap \Omega}\left(T Q+u_{t} u+v_{t} v\right)(T, x) d x \\
& +\frac{1}{\sqrt{2}} \int_{M_{T}^{S} \cap \Omega_{T}^{S}}\left(x \cdot P+t Q+u_{t} u+v_{t} v\right) d \sigma(x, t) \\
& -\int_{\partial \Omega_{T}^{S} \cap K_{T}^{S}} \vec{n}(x) \cdot(t P) d \tilde{\sigma}(x, t) \leq 0 .
\end{aligned}
$$

Using Hölder's inequality, we have

$$
\begin{aligned}
& \left|\int_{D_{S}}\left(S Q+u_{t} u+v_{t} v\right)(S, x) d x\right| \\
& \quad \leq \int_{D_{S}}|S||Q| d x+\int_{D_{S}}\left|u_{t} u\right| d x+\int_{D_{S}}\left|v_{t} v\right| d x \\
& \quad \leq|S| E\left(u, D_{S}\right)+\left\|u_{t}\right\|_{L^{2}\left(D_{S}\right)}\|u\|_{L^{2}\left(D_{S}\right)}+\left\|v_{t}\right\|_{L^{2}\left(D_{S}\right)}\|v\|_{L^{2}\left(D_{S}\right)} \\
& \quad \leq|S| E\left(u, D_{S}\right)+\left\|u_{t}\right\|_{L^{2}\left(D_{S}\right)}\|u\|_{L^{6}\left(D_{S}\right)}|S|^{1 / 3}+\left\|v_{t}\right\|_{L^{2}\left(D_{S}\right)}\|v\|_{L^{6}\left(D_{S}\right)}|S|^{1 / 3} \\
& \quad \leq C\left(|S| E\left(u, v, D_{S}\right)+|S|^{1 / 3} E\left(u, v, D_{S}\right)^{2 / 3}\right) \rightarrow 0 \text { as } S \nearrow 0^{-} .
\end{aligned}
$$

By letting $S \nearrow 0^{-}$, we see that

$$
\begin{aligned}
-\int_{D_{T} \cap \Omega}\left(T Q+u_{t} u+\right. & \left.v_{t} v\right)(T, x) d x \\
& +\frac{1}{\sqrt{2}} \int_{M_{T}^{0} \cap \Omega_{T}^{0}}\left(x \cdot P+t Q+u_{t} u+v_{t} v\right) d \sigma(x, t) \\
\leq & \int_{\partial \Omega_{T}^{0} \cap K_{T}^{0}} \vec{n}(x) \cdot(t P) d \tilde{\sigma}(x, t) .
\end{aligned}
$$

Next, we denote the two terms on the left hand side of the above inequality by $I+I I$, and repeating the arguments from [11, p. 3146 and equation (2.10)], we obtain

$$
I \geq-\frac{1}{2} \int_{\partial D_{T} \cap \Omega}\left(|u|^{2}+|v|^{2}\right) d S(x)-\frac{T}{2} \int_{D_{T} \cap \Omega}\left(\frac{1}{3}\left(|u|^{6}+|v|^{6}\right)+|u|^{2}|v|^{2}\right) d x,
$$




$$
\begin{aligned}
I I= & \frac{1}{\sqrt{2}} \int_{M_{T}^{0} \cap \Omega_{T}^{0}}\left(t\left|\frac{x}{|x|} \cdot \nabla u-u_{t}+\frac{u}{|x|}\right|^{2}+t\left|\frac{x}{|x|} \cdot \nabla v-v_{t}+\frac{v}{|x|}\right|^{2}\right) d \sigma \\
& +\frac{1}{2} \int_{\partial D_{T} \cap \Omega}\left(u^{2}+v^{2}\right)(T, x) d S(x) .
\end{aligned}
$$

Therefore, letting $S \nearrow 0^{-}$, we get

$$
\begin{aligned}
& |T| \int_{D_{T} \cap \Omega} \frac{1}{6}\left(|u|^{6}+|v|^{6}\right) d x \leq \int_{\partial \Omega_{T}^{0} \cap K_{T}^{0}} \vec{n}(x) \cdot(t P) d \tilde{\sigma}(x, t) \\
& \quad+|t| \frac{1}{\sqrt{2}} \int_{M_{T}^{0} \cap \Omega_{T}^{0}}\left(\left|\frac{x}{|x|} \cdot \nabla u-u_{t}+\frac{u}{|x|}\right|^{2}+\left|\frac{x}{|x|} \cdot \nabla v-v_{t}+\frac{v}{|x|}\right|^{2}\right) d \sigma .
\end{aligned}
$$

Notice that the second term can be bounded (see [11, p. 3146]) by

$$
|T| \operatorname{Flux}\left(u, v, M_{T}^{0}\right)+|T| \operatorname{Flux}\left(u, v, M_{T}^{0}\right)^{1 / 3} .
$$

Applying Dirichlet's boundary condition and the definition of $P$, we have on $\partial \Omega$

$$
\begin{aligned}
& \vec{n}(x) \cdot(t P) \\
= & \vec{n}(x) \cdot\left[-x \frac{\left|\nabla_{x} u\right|^{2}}{2}+\nabla_{x} u\left(x \cdot \nabla_{x}\right) u-x \frac{\left|\nabla_{x} v\right|^{2}}{2}+\nabla_{x} v\left(x \cdot \nabla_{x}\right) v\right] .
\end{aligned}
$$

Notice that

$$
\nabla_{x} u=\left(\vec{n}(x) \cdot \nabla_{x} u\right) \vec{n}(x)+\left(\vec{n}^{\perp} \cdot \nabla_{x} u\right) \vec{n}^{\perp}(x),
$$

where the tangential derivative $\left.\vec{n}^{\perp} \cdot \nabla_{x} u\right|_{\partial \Omega}$ equals 0 due to the Dirichlet condition. Therefore on $\partial \Omega$, we have $\nabla_{x} u=\left(\vec{n}(x) \cdot \nabla_{x} u\right) \vec{n}(x)$ and $\nabla_{x} v=$ $\left(\vec{n}(x) \cdot \nabla_{x} v\right) \vec{n}(x)$. As a consequence, the quantity on the right hand side of (4.2) is estimated by

$$
\frac{1}{2}(\vec{n}(x) \cdot x)\left|\frac{\partial u}{\partial \vec{n}}\right|^{2}+\frac{1}{2}(\vec{n}(x) \cdot x)\left|\frac{\partial v}{\partial \vec{n}}\right|^{2} .
$$

Consequently, we obtain

$$
\begin{aligned}
|T| \int_{D_{T} \cap \Omega} \frac{1}{6}\left(|u|^{6}+|v|^{6}\right) d x & \\
\leq & C \int_{\partial \Omega_{T}^{0} \cap K_{T}^{0}}\left(\frac{1}{2}(\vec{n}(x) \cdot x)\left|\frac{\partial u}{\partial \vec{n}}\right|^{2}+\frac{1}{2}(\vec{n}(x) \cdot x)\left|\frac{\partial v}{\partial \vec{n}}\right|^{2}\right) d \tilde{\sigma}(x, t) \\
& +|T| \operatorname{Flux}\left(u, v, M_{T}^{0}\right)+|T| \operatorname{Flux}\left(u, v, M_{T}^{0}\right)^{1 / 3} .
\end{aligned}
$$


For the same reason as in [2, p. 9], the first integral is bounded by

$$
\sup _{x \in K_{T}^{0}}|x|^{2} \int_{\partial \Omega_{T}^{0}}\left(\left|\frac{\partial u}{\partial \vec{n}}\right|^{2}+\left|\frac{\partial v}{\partial \vec{n}}\right|^{2}\right) d \varrho(x) d t \leq C|T|^{2} E(u, v),
$$

and, finally, we have

$$
\int_{D_{T} \cap \Omega} \frac{1}{6}\left(|u|^{6}+|v|^{6}\right) \leq C\left(|T| E(u, v)+\operatorname{Flux}\left(u, v, M_{T}^{0}\right)+\operatorname{Flux}\left(u, v, M_{T}^{0}\right)^{1 / 3}\right) .
$$

This means that the right hand side goes to 0 as $T \nearrow 0^{-}$. Therefore, we obtain (4.1). In the above calculations all integrals on $K_{T}^{0}$ and $M_{T}^{0}$ have to be understood as the limits as $S \rightarrow 0^{-}$of the integrals on $K_{T}^{S}$ and $M_{T}^{S}$.

We are in a position to show that solutions to $(1.3)-(1.5)$ are global in time, which will complete the proof of Theorem 1.1 .

From the local existence in Section 2, we continue to consider $(u, v)$ as the unique forward maximal solution to the system $(1.3)-(1.5)$ in the space $\mathcal{X}\left(\left[0, t_{0}\right)\right)$ with $t_{0}<+\infty$ (see Theorem 2.1). Taking a point $x_{0} \in \bar{\Omega}$, we aim at proving that $(u, v)$ can be extended in a neighborhood of $\left(x_{0}, t_{0}\right)$, which brings a contradiction. By translation invariance, $\left(x_{0}, t_{0}\right)$ can be moved to the space-time origin $(0,0)$.

We will work on localized space-time cones and perform estimates following [1] and [14]. For $t \leq t^{\prime} \leq 0$, we define the norms supported on small light cones by

$$
\begin{aligned}
& \|(u, v)\|_{\left(L^{p} ; L^{q}\right)\left(K_{t}^{t^{\prime}}\right)} \\
& \quad=\left(\int_{t}^{t^{\prime}}\left(\int_{D_{s} \cap \Omega}|u|^{q}(s, x) d x\right)^{p / q} d s\right)^{1 / p}+\left(\int_{t}^{t^{\prime}}\left(\int_{D_{s} \cap \Omega}|v|^{q}(s, x) d x\right)^{p / q} d s\right)^{1 / p} .
\end{aligned}
$$

We assume that $x_{0} \in \partial \Omega$, otherwise by Huygens' principle, the approach to global solution is similar to the Cauchy problems. For then $|t|$ can be chosen small enough so that the time-slice of the small light cone (with vertex $\left.\left(x_{0}, t_{0}\right)\right)$ is contained in the interior of $\Omega$. Moreover, for the slice with its radius enlarged by a small $\epsilon>0$, we can choose $|t|$ even smaller to let it shrink into the interior of $\Omega$. To this the standard argument applies (see [8], [11).

The following proposition is crucial in this section.

Proposition 4.1. For any $\varepsilon>0$, there is $t<0$ such that

$$
\|(u, v)\|_{\left(L^{5} ; L^{10}\right)\left(K_{t}^{0}\right)}<\varepsilon .
$$

Before proving this proposition, we need an extension lemma from [1].

Lemma 4.1. For any $x_{0} \in \bar{\Omega}$ there is $r_{0}>0$ such that for any $0<r<r_{0}$ and any $v \in H_{0}^{1}(\Omega) \cap L^{p}(\Omega)$ there exists a function $\tilde{v}_{r} \in H_{0}^{1}(\Omega)$ (independent 
of the choice of $1 \leq p \leq \infty)$ satisfying

$$
\begin{gathered}
\left.\left(\tilde{v}_{r}-v\right)\right|_{\left\{\left|x-x_{0}\right|<r\right\} \cap \Omega}=0, \\
\int_{\Omega}|\nabla \tilde{v}|^{2} \leq C \int_{\Omega}|\nabla v|^{2}, \quad\left\|\tilde{v}_{r}\right\|_{L^{p}(\Omega)} \leq C\|v\|_{L^{p}\left(\left\{\left|x-x_{0}\right|<r\right\}\right)} .
\end{gathered}
$$

In other words, one can extend functions in $H_{0}^{1} \cap L^{p}$ on the ball $\left\{\left|x-x_{0}\right|<r\right\}$ to functions in $H_{0}^{1}(\Omega) \cap L^{p}(\Omega)$ with uniform bounds with respect to small $r>0$ for the $H^{1}$ and the $L^{p}$ norms respectively.

Furthermore, for any $u \in L^{\infty}\left((-1,0) ; H_{0}^{1}(\Omega)\right) \cap L_{\mathrm{loc}}^{1}\left((-1,0) ; L^{p}(\Omega)\right)$, there exists a function $\check{u} \in L^{\infty}\left((-1,0) ; H_{0}^{1}(\Omega)\right) \cap L_{\text {loc }}^{1}\left((-1,0) ; L^{p}(\Omega)\right)$ satisfying (uniformly with respect to $t$ )

$$
\begin{gathered}
\left.(\check{u}-u)\right|_{\left\{\left|x-x_{0}\right|<-t\right\} \cap \Omega}=0, \\
\int_{\Omega}\left(|\nabla \check{u}|^{2}(t, x)+\left|\partial_{t} \check{u}\right|^{2}(t, x)\right) d x \leq C \int_{\Omega}\left(|\nabla u|^{2}(t, x)+\left|\partial_{t} u\right|^{2}(t, x)\right) d x, \\
\|\check{u}(t, \cdot)\|_{L^{p}(\Omega)} \leq C\|u(t, \cdot)\|_{L^{p}\left(\Omega \cap\left\{\left|x-x_{0}\right|<-t\right\}\right)} \quad \text { for a.e. } t \in(-1,0) .
\end{gathered}
$$

Proof of Proposition 4.1. Let $\breve{u}, \breve{v}$ be the functions given by Lemma 4.1. Then $(\check{u})^{5}$ is equal to $u^{5}$ on $K_{t}^{0} \cap \Omega_{t}^{0}$ for $t<t^{\prime}<0$. Applying the nonlinear estimate (2.2) to (2.5) obtained in Section 2 and employing (4.9), we have

$$
\begin{aligned}
& \left\|\check{u}^{5}+\check{v}^{2} \breve{u}\right\|_{L^{5 / 4}\left(\left(t, t^{\prime}\right) ; W^{7 / 10,5 / 4}(\Omega)\right)} \\
& \leq C M(u, v)\|(u, v)\|_{L^{\infty}\left(\left(t, t^{\prime}\right) ; H^{1}(\Omega)\right)}^{7 / 0}\|(u, v)\|_{\left(L^{\infty} ; L^{6}\right)\left(K_{t}^{0}\right)}^{3 / 10}, \\
& \left\|\check{v}^{5}+\check{u}^{2} \breve{v}\right\|_{L^{5 / 4}\left(\left(t, t^{\prime}\right) ; W^{7 / 10,5 / 4}(\Omega)\right)} \\
& \leq C M(u, v)\|(u, v)\|_{L^{\infty}\left(\left(t, t^{\prime}\right) ; H^{1}(\Omega)\right)}^{7 / 10}\|(u, v)\|_{\left(L^{\infty} ; L^{6}\right)\left(K_{t}^{0}\right)}^{3 / 10},
\end{aligned}
$$

where

$$
M(u, v):=\|(u, v)\|_{\left(L^{5} ; L^{10}\right)\left(K_{t}^{t^{\prime}}\right)}^{4}+\|(u, v)\|_{\left(L^{5} ; L^{10}\right)\left(K_{t}^{t^{\prime}}\right)}^{2} .
$$

Let $(\mu, \nu)$ be the solution to the system

$$
\begin{aligned}
\left(\partial_{s}^{2}-\Delta\right) \mu & =-|\check{u}|^{5}-|\check{v}|^{2} \check{u}, \\
\left(\partial_{s}^{2}-\Delta\right) \nu & =-|\check{v}|^{5}-|\check{u}|^{2} \check{v},
\end{aligned}
$$

subject to the boundary conditions

$$
\left.\mu\right|_{\partial \Omega}=\left.\nu\right|_{\partial \Omega}=0
$$

with initial values at $s=t$,

$$
\left.(\mu-u)\right|_{s=t}=\left.(\nu-v)\right|_{s=t}=0,\left.\quad \partial_{s}(\mu-u)\right|_{s=t}=\left.\partial_{s}(\nu-v)\right|_{s=t}=0 .
$$

By Huygens' principle (for an inhomogeneous version see [10], [18]), $(\mu, \nu)$ coincides with $(u, v)$ on $K_{t}^{0}$.

Applying Strichartz's inequality, Sobolev's imbedding and 4.3), 4.4, we get 


$$
\begin{aligned}
\|(u, v)\|_{\left(L^{5} ; L^{10}\right)\left(K_{t}^{t^{\prime}}\right)} \leq\|(\mu, \nu)\|_{L^{5}\left(\left(t, t^{\prime}\right) ; L^{10}(\Omega)\right)} \\
\leq C\|(\mu, \nu)\|_{L^{5}\left(\left(t, t^{\prime}\right) ; W_{0}^{3 / 10,5}(\Omega)\right)} \\
\leq C E(u, v, t)+C\left(\|(u, v)\|_{\left(L^{5} ; L^{10}\right)\left(K_{t}^{t^{\prime}}\right)}^{4}+\|(u, v)\|_{\left(L^{5} ; L^{10}\right)\left(K_{t}^{t^{\prime}}\right)}^{2}\right) \\
\quad \times\|(u, v)\|_{L^{\infty} H^{1}(\Omega)}^{7 / 10}\|(u, v)\|_{\left(L^{\infty} ; L^{6}\right)\left(K_{t}^{0}\right)}^{3 / 10}
\end{aligned}
$$

By (4.1), conservation of energy and continuity of the map

$$
t^{\prime} \in[t, 0) \mapsto\|(u, v)\|_{\left(L^{5} ; L^{10}\right)\left(K_{t}^{t^{\prime}}\right)}
$$

which takes value 0 when $t^{\prime}=t$, there exists a $t<0$ such that for any $t^{\prime} \in(t, 0)$ we have

$$
\|(u, v)\|_{\left(L^{5} ; L^{10}\right)\left(K_{t}^{t^{\prime}}\right)} \leq 2 C E(u, v)(t) .
$$

Letting $t^{\prime} \rightarrow 0$, we have

$$
\|(u, v)\|_{\left(L^{5} ; L^{10}\right)\left(K_{t}^{0}\right)} \leq 2 C E(u, v)(t) .
$$

Therefore, with any $\varepsilon>0$, taking $|t|$ small enough, we get

$$
\|(u, v)\|_{\left(L^{5} ; L^{10}\right)\left(K_{t}^{0}\right)} \leq \varepsilon,
$$

which completes the proof of Proposition 4.1.

Global existence and proof of Theorem 1.1. Supposing the lifespan of the solution is finite, we localize $(u, v)$ in a small backward space-time light cone and split $(u, v)$ into two parts corresponding to the solutions of a homogeneous equation with nonzero initial data and an inhomogeneous equation with vanishing initial data. We use different techniques to estimate those quantities and eventually, we find that their localized energy is small. Combining them, we see that the localized energy of $(u, v)$ is small. Then the dominated convergence theorem implies that the localized energy is still small with the radius of the base time-slice enlarged by a small quantity. The previous facts are employed together with the compactness of the domain to get the boundedness of the $L^{5}\left([-1,0] ; L^{10}(\Omega)\right)$ norm for $(u, v)$. Finally, we get a contradiction by Strichartz's estimate, Duhamel's formula and local existence theory. Let us state all these facts in a more precise way.

Let $t<t_{0}=0$ be sufficiently close to zero and let $(\xi, \eta)$ solve the linear system

$$
\begin{aligned}
& \left(\partial_{s}^{2}-\Delta\right) \xi=0 \\
& \left(\partial_{s}^{2}-\Delta\right) \eta=0
\end{aligned}
$$

subject to the boundary conditions

$$
\left.\xi\right|_{\partial \Omega}=\left.\eta\right|_{\partial \Omega}=0
$$

$$
\left.(\xi-u)\right|_{s=t}=\left.(\eta-v)\right|_{s=t}=0,\left.\quad \partial_{s}(\xi-u)\right|_{s=t}=\left.\partial_{s}(\eta-v)\right|_{s=t}=0 .
$$


Then the differences $\chi=u-\xi$ and $\zeta=v-\eta$ satisfy

$$
\begin{aligned}
& \left(\partial_{s}^{2}-\Delta\right) \chi=-u^{5}-v^{2} u \\
& \left(\partial_{s}^{2}-\Delta\right) \zeta=-v^{5}-u^{2} v \\
& \left.\chi\right|_{\partial \Omega}=\left.\zeta\right|_{\partial \Omega}=0 \\
& \left.(\chi, \zeta)\right|_{s=t}=(0,0)=\left.\left(\partial_{s} \chi, \partial_{s} \zeta\right)\right|_{s=t} .
\end{aligned}
$$

For the functions $\check{u}, \check{v}$ given by Lemma 4.1, we have

$$
\begin{aligned}
\|(\check{u}, \check{v})\|_{L^{5}\left((t, 0) ; L^{10}(\Omega)\right)} & \leq C \varepsilon, \\
\|(\check{u}, \check{v})\|_{L^{\infty}\left((t, 0) ; H_{0}^{1}(\Omega)\right)} & \leq C E(u, v)(t) .
\end{aligned}
$$

Let $(\tilde{\chi}, \tilde{\zeta})$ solve

$$
\begin{aligned}
& \left(\partial_{s}^{2}-\Delta\right) \tilde{\chi}=-\check{u}^{5}-\check{v}^{2} \check{u} \\
& \left(\partial_{s}^{2}-\Delta\right) \tilde{\zeta}=-\check{v}^{5}-\check{u}^{2} \check{v} \\
& \left.\tilde{\chi}\right|_{\partial \Omega}=\left.\tilde{\zeta}\right|_{\partial \Omega}=0 \\
& \left.(\tilde{\chi}, \tilde{\zeta})\right|_{s=t}=(0,0)=\left.\left(\partial_{s} \tilde{\chi}, \partial_{s} \tilde{\zeta}\right)\right|_{s=t}
\end{aligned}
$$

By Huygens' principle, $(\tilde{\chi}, \tilde{\zeta})$ and $(\chi, \zeta)$ coincide in $K_{t}^{0}$. On the other hand, using Proposition 2.1 and observing that

$$
\left\|a^{2} b\right\|_{L^{2}(\Omega)} \leq\|a\|_{L^{8}(\Omega)}^{2}\|b\|_{L^{4}(\Omega)} \leq 2 C\left(\|a\|_{L^{10}}^{4}+\|b\|_{L^{10}(\Omega)}^{2}\right),
$$

we get

$$
\begin{aligned}
& \|(\tilde{\chi}, \tilde{\zeta})\|_{L^{\infty}\left((t, 0) ; H_{0}^{1}(\Omega)\right)}+\left\|\partial_{s}(\tilde{\chi}, \tilde{\zeta})\right\|_{L^{\infty}\left((t, 0) ; L^{2}(\Omega)\right)}+\|(\tilde{\chi}, \tilde{\zeta})\|_{L^{5}\left((t, 0) ; W^{3 / 10,5}(\Omega)\right)} \\
& \quad \leq C\left(\left\|\check{u}^{5}+\check{v}^{2} \check{u}\right\|_{L^{1}\left((t, 0) ; L^{2}(\Omega)\right)}+\left\|\check{v}^{5}+\check{u}^{2} \check{v}\right\|_{L^{1}\left((t, 0) ; L^{2}(\Omega)\right)}\right) \\
& \quad \leq C\left(\|(\check{u}, \check{v})\|_{\left.L^{5}\left((t, 0) ; L^{10} \Omega\right)\right)}^{5}+\left\|\check{v}^{2} \check{u}\right\|_{L^{1}\left((t, 0) ; L^{2}(\Omega)\right)}+\left\|\check{u}^{2} \check{v}\right\|_{L^{1}\left((t, 0) ; L^{2}(\Omega)\right)}\right) \\
& \quad \leq C\left(\|(\check{u}, \check{v})\|_{\left.L^{5}\left((t, 0) ; L^{10} \Omega\right)\right)}^{5}+\|(\check{u}, \check{v})\|_{L^{5}\left((t, 0) ; L^{10}(\Omega)\right)}^{4}+\|(\check{u}, \check{v})\|_{L^{5}\left((t, 0) ; L^{10}(\Omega)\right)}^{2}\right)
\end{aligned}
$$

By (4.8), we can take $t<0$ close to 0 such that the right hand side of (4.9) is bounded by $C \varepsilon$. Finally, for any ball $B=B(0, r)$ centered at 0 with radius $r$, let

$E(f(s, \cdot), g(s, \cdot), B)=\int_{B \cap \Omega}\left(\left|\nabla_{s, x} f\right|^{2}+\left|\nabla_{s, x} g\right|^{2}+|f|^{2}|g|^{2}+\frac{|f|^{6}+|g|^{6}}{3}\right)(s) d x$.

Since $(\xi, \eta)$ solves the linear problem $4.5-4.7$, it follows that

$$
E(\xi(s, \cdot), \eta(s, \cdot), B(0,-s)) \rightarrow 0 \quad \text { as } s \nearrow 0^{-} .
$$

Moreover, for $s \nearrow 0^{-}$, we have

$$
E(\tilde{\chi}(s, \cdot), \tilde{\zeta}(s, \cdot), B(0,-s))<\varepsilon .
$$


Recalling $(u, v)=(\xi, \eta)+(\check{\chi}, \check{\zeta})$ in $K_{t}^{0}$, using $(4.9-4$ - 4.11) together with Sobolev's embedding $H_{0}^{1}(\Omega) \hookrightarrow L^{6}(\Omega)$ and Hölder's inequality, we deduce that there exists $s \nearrow 0^{-}$such that

$$
E(u(s, \cdot), v(s, \cdot), B(0,-s))<\varepsilon .
$$

However, since $\left(u, \partial_{s} u\right)$ and $\left(v, \partial_{s} v\right)$ are in $H_{0}^{1}(\Omega) \times L^{2}(\Omega)$, we have, by the dominated convergence theorem, for some small $\alpha>0$,

$$
E(u(s, \cdot), v(s, \cdot), B(0,-s+\alpha)) \leq 2 \varepsilon .
$$

According to $(3.2)$, on $\left\{\left|x-x_{0}\right|<-s^{\prime}+\alpha\right\}, s \leq s^{\prime}<0$, we also have

$$
\int_{B\left(0, \alpha-s^{\prime}\right)}\left(|u|^{6}+|v|^{6}\right) d x \leq 2 \varepsilon
$$

By the same argument as in the proof of Proposition 4.1 at the beginning of this section, the $\left(L^{5} ; L^{10}\right)(K)$ norm of the solution remains bounded on the truncated cone

$$
K=\left\{\left(x, s^{\prime}\right):\left|x-x_{0}\right|<\alpha-s^{\prime}, s<s^{\prime}<0\right\} \cap \Omega_{s}^{0} .
$$

This is true for all $x_{0} \in \bar{\Omega}$. By the compactness of $\overline{\Omega_{s}^{0}}$ and the finite covering, we get

$$
\|(u, v)\|_{L^{5}\left((s, 0) ; L^{10}(\Omega)\right)}<\infty .
$$

Consequently, by Duhamel's formula, the limits

$$
\lim _{s^{\prime} \rightarrow 0^{-}}\left(u, \partial_{s} u\right)\left(s^{\prime}, \cdot\right), \quad \lim _{s^{\prime} \rightarrow 0^{-}}\left(v, \partial_{s} v\right)\left(s^{\prime}, \cdot\right)
$$

exist in $H_{0}^{1}(\Omega) \times L^{2}(\Omega)$. Therefore, $(u, v)$ can be extended for small enough $s^{\prime}>0=t_{0}$ by the local existence argument from Section 2 .

Acknowledgements. The author thanks the refree for their carefull reading of this paper. Their dedication improved this work so much in both pointing out of some typing mistakes and making narration more clear.

\section{References}

[1] N. Burq, G. Lebeau and F. Planchon, Global existence for energy critical waves in 3-D domains, J. Amer. Math. Soc. 21 (2008), 831-845.

[2] N. Burq and F. Planchon, Global existence for energy critical waves in 3-D domains: Neumann boundary conditions, Amer. J. Math. 131 (2009), 1715-1742.

[3] J. Chazarain and A. Piriou, Introduction to the Theory of Linear Partial Differential Equations, Stud. Math. Appl. 14, North-Holland, Amsterdam, 1982.

[4] M. Christ and A. Kiselev, Maximal functions associated to filtrations, J. Funct. Anal. 179 (2001),409-425.

[5] E. B. Davies, Spectral Theory and Differential Operators, Cambridge Univ. Press, 1995.

[6] J. Ginibre and G. Velo, The global Cauchy problem for the nonlinear Klein-Gordon equation, Math. Z. 189 (1985), 487-505. 
[7] J. Ginibre and G. Velo, The global Cauchy problem for the nonlinear Klein-Gordon equation II, Ann. Inst. H. Poincaré Anal. Non Linéaire 6 (1989), 15-35.

[8] M. Grillakis, Regularity and asymptotic behaviour of the wave equation with a critical nonlinearity, Ann. of Math. 132 (1990), 485-509.

[9] K. Jörgens, Das Anfangswertproblem im Grossen für eine Klasse nichtlinearer Wellengleichungen, Math. Z. 77 (1961), 295-308.

[10] C. Miao, Modern Methods for Nonlinear Wave Equations, 2nd ed., Monogr. Modern Pure Math. 133, Sci. Press, Beijing, 2010.

[11] C. Miao and Y. Zhu, Global smooth solutions for a nonlinear system of wave equations, Nonlinear Anal. 67 (2007), 3136-3151.

[12] J. Rauch, The $u^{5}$ Klein-Gordon equation. II. Anomalous singularities for semilinear wave equations, in: Nonlinear Partial Differential Equations and Their Applications, Collège de France Seminar, Vol. I (Paris, 1978/1979), Res. Notes in Math. 53, Pitman, Boston, MA, 1981, 335-364.

[13] I. Segal, Non-linear partial differential equations in quantum field theory, in: Proc. Sympos. Appl. Math. 17, Amer. Math. Soc., 1965, 210-226.

[14] J. Shatah and M. Struwe, Regularity results for nonlinear wave equations, Ann. of Math. 138 (1993), 503-518.

[15] —, -, Well-posedness in the energy space for semilinear waves equations with critical growth, Int. Math. Res. Notices 1994, no. 7, 303-309.

[16] H. Smith and C. D. Sogge, On the critical semilinear wave equation outside convex obstacles, J. Amer. Math. Soc. 8 (1995), 879-916.

[17] - - - On the $L^{p}$ norm of spectral clusters for compact manifolds with boundary, Acta Math. 198 (2007), 107-153.

[18] C. D. Sogge, Lectures on Nonlinear Wave Equations, 2nd ed., Int. Press Publ., 2008.

[19] M. Struwe, Globally regular solutions to the $u^{5}$ Klein-Gordon equation, Ann. Scuola Norm Sup. Pisa 15 (1988), 495-513.

Jianwei Yang

The Graduate School of China Academy of Engineering Physics

P.O. Box 2101

Beijing 100088, P.R. China

E-mail: geewey.young@gmail.com

Received on 17.3.2011;

revised version on 4.4 .2011 